$\underline{\text { DOSSIER }}$

\title{
Usuarios de nuevos medios, Comunicación e interacción social
}

\section{Resumen}

El artículo plantea que los nuevos medios promueven la satisfacción de las crecientes necesidades de comunicación de la sociedad contemporánea, realizándose una breve descripción de las modalidades de interacción que suponen para los usuarios. Se formula un modelo de la interacción de los individuos en los medios sociales.

Palabras clave: : Interacción - Medios sociales - Teléfonos Móviles.

\section{Users of New Media,} Communication and social interaction

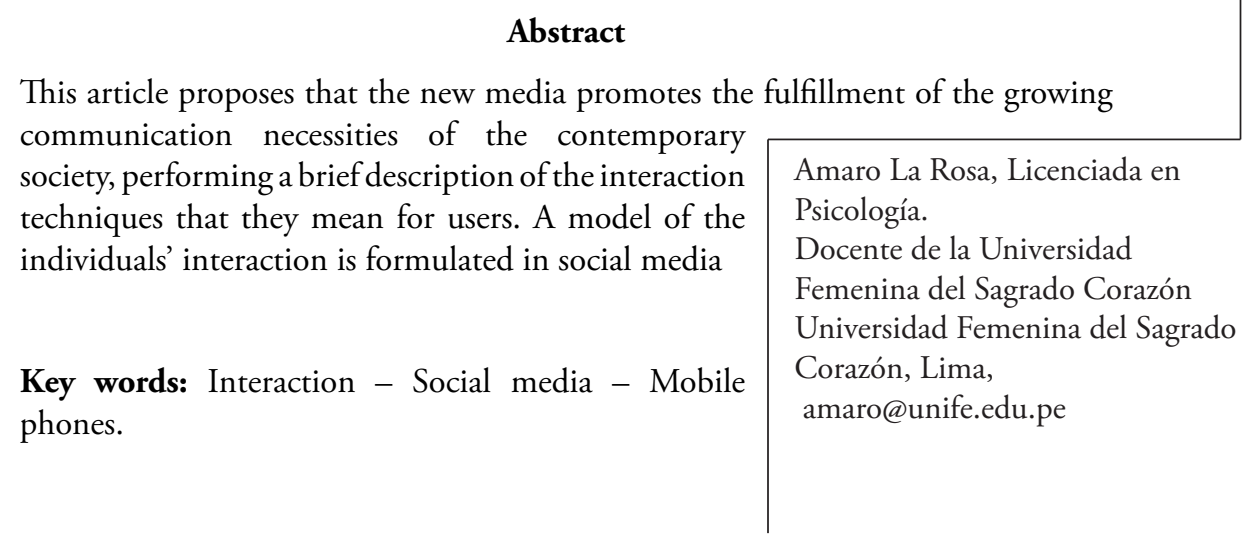


El escenario es una clase universitaria en cualquier lugar del mundo. Podemos suponer que todos los estudiantes están atendiendo al catedrático mientras explica un tema, pero esta no es la verdad. En contra de las normas establecidas algunos de ellos están recibiendo y enviando mensajes de texto o revisando su cuenta de un medio social, a través de sus teléfonos móviles. ¿Qué pasa con ellos? ¿Están más interesados en la comunicación virtual para el entretenimiento que en el tema de la clase? ¿Tienen acaso la urgente necesidad de interactuar en ese mismo momento con sus contactos? ¿Temen acaso perder sus relaciones interpersonales online por no responder inmediatamente?

No requiere constatación empírica la presunción de que necesitamos ser escuchados por los demás, lo que es indudablemente parte de la naturaleza del ser humano, en esencia gregario. Las condiciones de vida en la sociedad contemporánea facilitan múltiples contactos superficiales. Así, podemos observar aquí lo que Merton denomina rasgos anómicos, que en este caso involucra la disminución de los contactos humanos efectivos, cara a cara y el incremento de las necesidades de comunicación que en la práctica no son satisfechas. Hemos observado que estas necesidades son tanto más importantes por los déficit de comunicación y las grandes distancias en las urbes, que propician la soledad en las personas. Desde esta perspectiva, en el ciberespacio se convierte en realidad la satisfacción de la necesidad de diálogo, de interacción que requerimos en la vida diaria. Pero, en términos reales, no se trata, en este caso, de una comunicación directa, cara a cara, porque es posible solamente con la intermediación de recursos tecnológicos /computadoras, teléfonos móviles, etc.), que nos permiten establecer y mantener el contacto con otros seres humanos.

Los medios digitales que son parte importante de la vida cotidiana de millones de personas alrededor del mundo, especialmente en los entornos urbanos y crecientemente en los rurales, están modificando el sistema de comunicación humana (Mora, 2012). El acceso a estos medios, con los innumerables recursos que podemos encontrar ahora en el mercado global, desde el desarrollo de la web 2.0, los convierte en instrumentos para optimizar las relaciones con personas de diversos contextos. Tal como veremos en el presente trabajo, esto se transforma involucrando el uso de diversos instrumentos tecnológicos, conservando la percepción de presencia en el contexto común y en la interacción con los pares. 
Para Lister, Dovey, Giddings, Grant \& Kelly (2009, pp. 12-13) los nuevos medios involucran:

1. Nuevas experiencias textuales.

2. Nuevas maneras de representar al mundo.

3. Nuevas relaciones entre los sujetos (usuarios y consumidores) y las tecnologías.

4. Nuevas experiencias de las relaciones corporalidad, identidad y comunidad.

5. Nuevas concepciones de la relación biológica del cuerpo con los medios tecnológicos.

6. Nuevos patrones de organización y producción.

Rasmussen (1997, p. 9) anota que "las tecnologías de comunicación, a diferencia de los mass media, "combinan las situaciones de producción de la conversación, con la conversación en sí misma”. Esta idea puede ser actualizada ahora para entender el caso de los nuevos medios en los cuales la persona produce e intercambia conversaciones o información mediante una rica y vívida experiencia de interacción la cual incrementa la popularidad de estos recursos. La audiencia, en este caso, es eminentemente activa y las personas permanecen usualmente en una actitud de "en búsqueda de..." cuando son usuarios de correo electrónico, chat, grupos de interés, motores de búsqueda o los social media e interactúan con otras personas, "en algún lugar en el ciberespacio".

Este criterio coincide con el de Zuluaga (2012, p.241) cuando afirma que "son ciudadanos-conectados que han perdido su lugar de súbditos y una actitud pasiva, para ganar la diversidad expresiva"

Lemos (2007, p.41) las denomina redes sociales de establecimiento de relaciones de Internet, y, a su entender, su misión básica es la interacción social, esto es, conectar personas y comunicarlas entre sí.

Presentamos un modelo preliminar de interacción en social media (Gráfico 1). Desde luego, en el gráfico, los elementos tienen una apariencia estática, pero en el mundo real desarrollan un proceso constante de interacción. Las personas no están aisladas, pues interactúan con otras en la misma u otras redes sociales. y en cada persona $(\mathrm{P})$ del lado derecho detectaríamos la influencia de los mismos factores observados en la persona del lado izquierdo. 


\section{GRÁFICO 1}

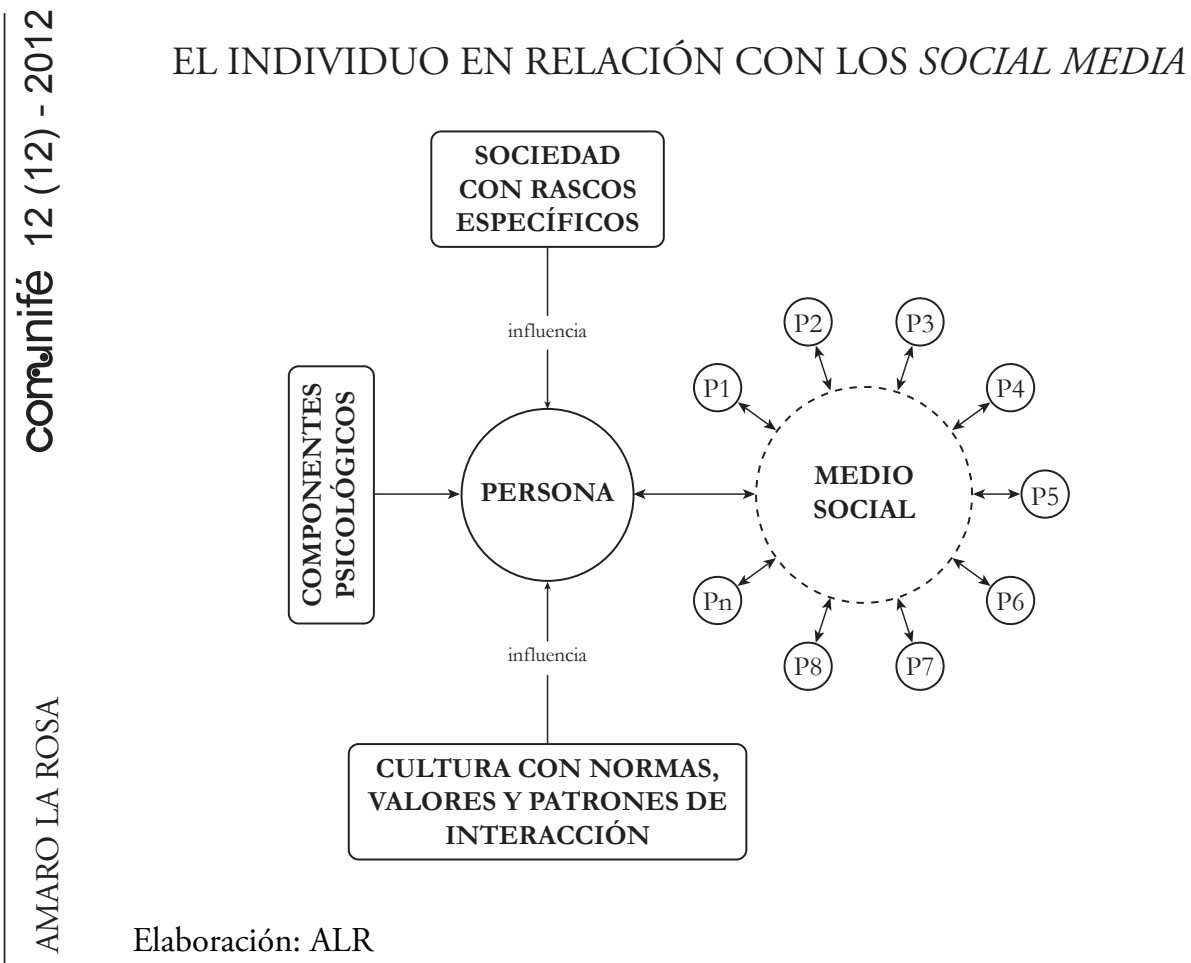

Es este un modelo general acerca de los medios sociales. Naturalmente, reconocemos que hay rasgos específicos y diferentes recursos en cada clase de red, que encontraremos y manejamos durante nuestras rutinas cotidianas en el ciberespacio. En la práctica, la situación es bastante más compleja por lo que a partir de esta constatación presentamos y discutimos este modelo en una serie de foros nacionales e internacionales, recibiendo comentarios y sugerencias de los colegas. Algunos de estos criterios se han tomando en cuenta para la construcción del modelo actualizado (Gráfico 2). 
GRÁFICO 2

\section{UN MODELO DE RELACIONES INTERPERSONALES EN LOS MEDIOS SOCIALES}

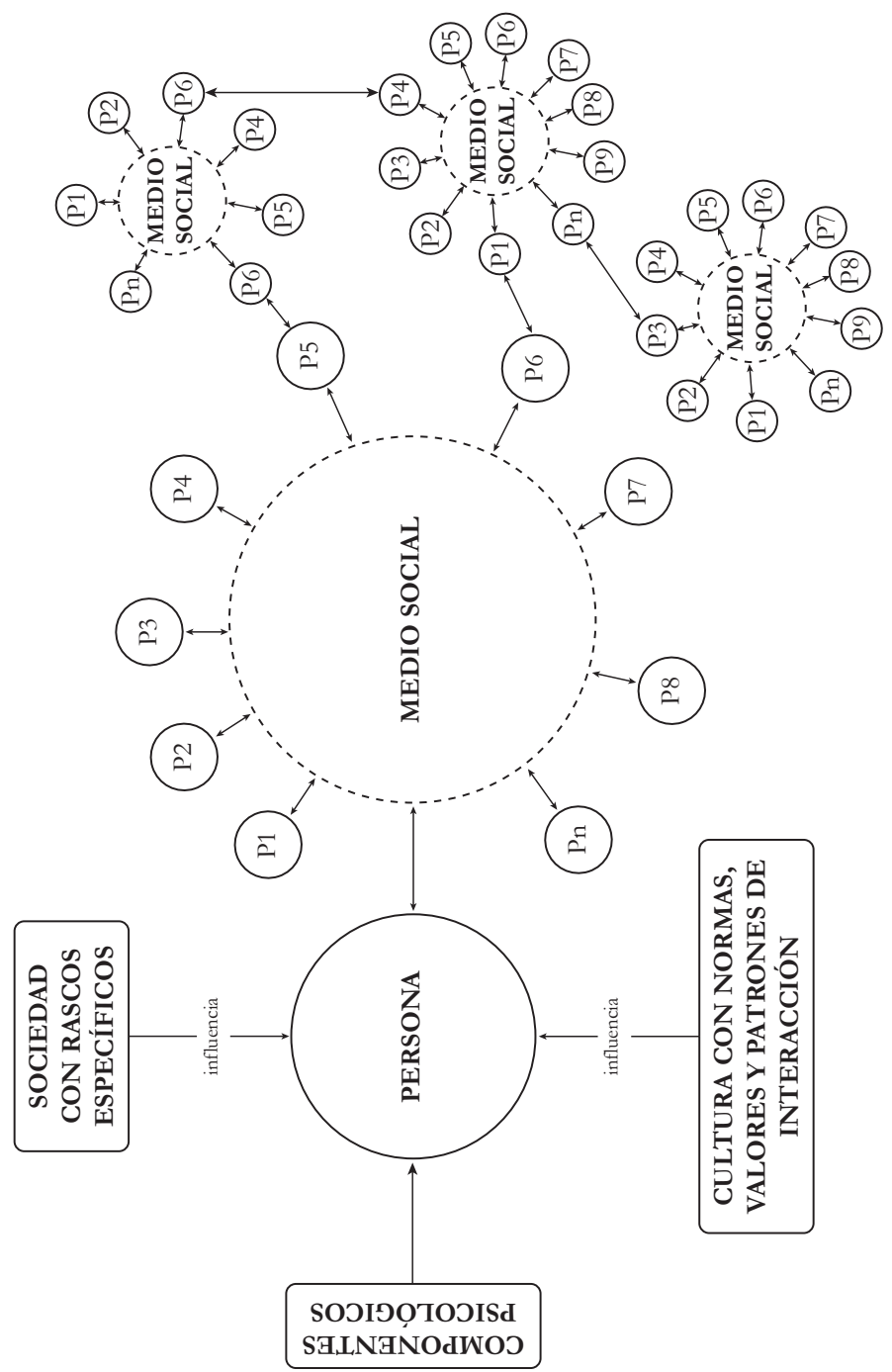

Elaboración: ALR 
Tal como apreciamos, cada individuo construye sus propias redes sociales, convirtiéndose en un nodo de un complejo conjunto en el mare magnum de los medios sociales. Por ello, probablemente, la mejor forma de representar esta realidad sea en un complejo modelo tridimensional, que intentaremos desarrollar en el futuro. Desde luego, un modelo es una representación de la realidad, que por su propia naturaleza se refiere solamente a un aspecto de la misma. Es bien cierto que cualquier individuo está integrado en diversas redes, no utiliza necesariamente todas ellas en la misma dimensión y tampoco está suscrito a las mismas redes que los demás.

Entre los medios cuyo uso ha crecido más aceleradamente están los teléfonos móviles que tal como lo revela Clonen (2002) no constituyen solamente recursos sino que son herramientas tecnológicas que se han internalizado en los complejos patrones de interacción y comunicación, contribuyendo al desarrollo de modalidades particulares de intercambio simbólico. En este sentido, para Oskman \& Turtiainen (2004), desde el desarrollo intensivo de los teléfonos móviles en Finlandia, los adolescentes han generado y puesto en vigencia una cultura de comunicación e interacción la cual incorpora, entre otros rasgos, los mensajes de texto y los recursos multimedia que sirven para articular su espacio personal y su identidad. Podemos afirmar, sin lugar a dudas, que esta constituye una cultura de carácter relativamente universal entre los jóvenes de las zonas urbanas del mundo.

En su análisis de los patrones de comunicación cotidiana de los adolescentes, usuarios de teléfonos móviles, Thulin \& Vihelmson (2007) enfocan su atención en tres áreas de implicaciones potenciales:

- Patrones de contacto e interacción cara a cara.

- Otras formas de movilidad especial.

- Planificación individual y uso del tiempo.

Estos autores encuentran que la interacción total con su entorno social se incrementa, al menos en esta etapa de la vida. Similarmente, observamos en nuestra práctica cotidiana, que los jóvenes tienden a permanecer en constante comunicación. Así, apenas dejan de verse, continúan comunicándose frecuentemente vía SMS y cuando arriban a su casa se dirigen a su computadora y continúan con la interacción 
usando diferentes recursos tales como Messenger (desaparecido en marzo de 2013), Facebook, Twitter o Skype, entre otros. Desde la perspectiva de Baym (2009), las relaciones interpersonales son crecientemente multimodales y se emplean más recursos de comunicación cuanto más cercanos sean los lazos entre los individuos.

Es claro que los jóvenes ven a Internet como una gran oportunidad para ampliar sus horizontes y contactarse con personas del mundo entero, y que se les denomina nativos digitales por haber nacido en mundo donde Internet y la comunicación digital son una realidad. Sin embargo, Madden (2010) reporta que el uso de los medios sociales crece rápidamente entre adultos de mediana edad, quienes son migrantes digitales. La investigación revela que entre abril de 2009 y mayo de 2010, en adultos de 50-64 años, su uso creció en 88 por ciento; y para los mayores de 65 años o más, en 100 por ciento.

El nivel de popularidad que experimentamos en la vida diaria, esto es, la evidencia de ser percibido como una persona valiosa por nuestros amigos y la sociedad, puede ser comprendida en vinculación con la importancia relativa del individuo para su comunidad y sus pares cercanos. No obstante, en el mundo de los medios sociales tiene más prestigio quien cuenta con el mayor número de contactos agregados a su cuenta. Un asunto clave para la consolidación de las redes es la mutua confianza entre las personas, la cual se relaciona directamente con la posibilidad de percibir al otro e incluirlo en su matriz de referencia. En este sentido, con las redes sociales online las zonas de percepción de proximidad no tienen limitaciones de tiempo ni espacio real. La cercanía está dada en función de la participación. Así, si alguien recibe un like (me gusta) en Facebook siente que su mensaje ha sido captado y aceptado por alguien, a quien a veces curiosamente no conoce en realidad.

Para los usuarios de los nuevos medios, los espacios públicos y privados no se interfieren. Una persona puede compartirlos sin problemas, pues en su mentalidad han cambiado los propios espacios de interacción. Los espacios públicos y privados se han transformado en espacios híbridos que integran a las personas que usan teléfonos móviles, los cuales, en función de la convergencia de las telecomunicaciones y de la digitalización, están ahora conectadas a Internet y a los medios sociales. 
Los espacios son lugares de interacción en los cuales los usuarios están en contacto utilizando sus teléfonos móviles. En un reciente artículo (La Rosa, 2011) describimos algo observado, al menos en la sociedad peruana: La estrecha relación entre la edad y la privacidad en la comunicación móvil. Los jóvenes tienen una mayor apertura para hablar acerca de su vida privada mediante sus teléfonos móviles, bajo el criterio de que es su propia decisión hablar o no sobre determinado asunto y pensando que sus vecinos están en otro espacio. La comunicación virtual permite la construcción de una suerte de burbuja, de un espacio personal virtual en el espacio público.

Schneider, Feldmann, Krishnamurthy \& Willinger (2009), en un estudio sobre usuarios de medios sociales que utilizaban dos proveedores de servicios de Internet, con 20,000 usuarios de líneas dedicadas cada uno, encontraron que la mayoría de las sesiones en las cuales participaban eran cortas (poco minutes) y pequeñas en términos de volumen y que las sesiones largas, estaban dominadas por periodos de inactividad en los cuales los usuarios no interactuaban.

Las conexiones humanas en los medios sociales son distintas que las producidas de modo offline porque la interacción entre los participantes usualmente se expandía hacia personas que en el momento no se conocían unas a las otras (Vesnić-Alujević (2012). Pero esto no significa que no existan relaciones entre estos dos tipos de redes. De acuerdo con Goodings, Locke \& Brown (2007, pp.475-476) las formas de comunidades mediadas que suponen los medios sociales, mantienen simultáneamente relaciones complejas y de diversos matices con modalidades de comunicación online y offline.

La investigación de Antoci, Sabatini y Sodini (2010) revela que las personas están adoptando sitios de social media como un entorno de suma importancia para el desarrollo de relaciones interpersonales por dos razones:

- La reducción del tiempo libre para los encuentros cara a cara.

- Las crecientes oportunidades de participación surgidas con los nuevos medios. 
Graybill (2010) encuentra que a los participantes de su investigación realizada con agricultores sudafricanos les agrada utilizar Facebook para comunicarse y compartir historias, anotando que constituye un efectivo canal de comunicación para generar conciencia e incrementar el número de afiliados. El constante involucramiento y la motivación subyacente constituyen factores clave en el éxito de Facebook en esta población. De modo similar para Barona y Cabezas (2011), Facebook facilita la interacción entre los usuarios al posibilitar una comunicación directa y en tiempo real.

Tomando como base a la teoría de usos y gratificaciones, Martínez (2010) estima la existencia de un conjunto de valores positivos que parecen estar "desprendiendo" las redes sociales y que constituyen variables importantes que motivan la interacción:

- Confianza

- Amistad

- Compañía

- Felicidad

- Cultura

La investigación de Cloete (2010) señala que Facebook es usada fundamentalmente para la comunicación, detectándose como factores de mayor importancia: Eficiencia, velocidad, simplicidad, conveniencia y comodidad.

Pero, ¿qué pasa con aquellas personas quienes están aisladas de su familia y amigos y quienes dedican gran parte de sus momentos libres a la comunicación online con otros pares? En este caso, ingresamos de nuevo a un terreno sobre el cual hemos caminado reiteradamente, dando algunas veces pasos en falso y sobre el cual se han escrito diversos puntos de vista. La tecnología no es la culpable del aislamiento del individuo; es la misma persona quien ya tenía problemas previos en su comportamiento que dificultaban la comunicación interpersonal, antes de la presencia de la tecnología.

La vida cotidiana ya no es la misma desde el surgimiento y difusión de Internet y de los nuevos medios en general, pero en contra de los contemporáneos ludditas, pensamos que forman parte de la natural y permanente evolución de la sociedad humana, más cerrada y local en el 
pasado, más abierta y globalmente interconectada ahora y en el futuro. Encontraremos, en los actuales sistemas de comunicación, las profundas huellas del largo recorrido de los recursos tecnológicos desarrollados a lo largo de la historia de la humanidad para satisfacer las crecientes necesidades de comunicación del ser humano; incrementadas notoriamente ahora en la era que conocemos como la Sociedad de la información.

Referencias

Antocu, A., Sabatini, F, \& Socini, M. (2010) See you on Facebook: The effect of social networking on human interaction MPR Research Paper No 27661 München: Münich Personal RePEc Ärchive. Revisado: Julio 20 de 2012. Disponible: http://mpra.ub.uni-muenchen.de/27661/

Barona, M. y Cabezas, J. (2011) Redes Sociales Virtuales y su incidencia en los procesos de comunicación de los Movimientos Sociales indígena y ecologista del Ecuador. Tesis Licenciado, Carrera De Ciencias de la Comunicación, Universidad Politécnica del Ecuador, Quito.

Baym, N. (2009) A call for grounding in the face of blurred boundaries Journal of Computer-Mediated Communication, Volume 14 (3) 720-723

Cloete, A. (2010) Exploring the uses and gratifications of Facebook: A psychological study. Thesis MA, Faculty of Humanities. University of Pretoria

Clonen, K (2002) SMS Behavior of Flemish Juveniles: Cell Phone Usage as an Instrument of Social Bonding and Peer Group Prestige Ethnographica Vol 2, 1-9. Revisado; Setiembre 2 de 2012. Disponible:

http://soc.kuleuven.be/antropologie/ethnographica/2002/Clonen.pdf Goodings, L, Locke, A \& Brown, S. (2007) Social Networking Technology: Place and Identity in Mediated Communities Journal of Community \& Applied Social Psychology, 17, 463-476 
Graybill, M. (2010) Exploring the use of Facebook as a communication tool in agricultural-related social movements. Thesis M.S., Graduate Faculty of Texas Tech University

La Rosa (2011) Teléfonos móviles: "el secreto mundo privado en el espacio público" Tesis Psicológica, 6, 74-89

Lemos, A. (2007) Desenvolvimento moral e uso de redes sociais de relacionamento na Internet: Uma relaçao possivel? Dissertaçáo de Mestrado, Programa de Pós-Graduaçáo em Psicologia da Universidade Federal de Minas Gerais.

Lister, M., Dovey, J., Giddings, S., Grant, I. \& Kelly, K. (2009) New media: A critical introduction New York: Routledge

Martínez, F. (2010) La teoría de los usos y gratificaciones aplicada a las redes sociales. Ponencia presentada en el II Congreso Internacional Comunicación 3.0, Salamanca, Octubre. Revisado: agosto 18 de 2012. Disponible:

http://campus.usal.es/ - comunicacion3punto0/comunicaciones/087. pdf

Mora, J. (2012) Medios interactivos y cultura digital: Alfabetización hipermedia en Perú y Bolivia, Comunicar, Vol XX, 39, 139-149

Oksman, V. \& Turtiainen, J. (2004) Mobile Communication as a Social Stage: Meanings of Mobile Communication in Everyday Life among Teenagers in Finland New Media \& Society, vol. 6, 3: pp. 319-339

Rasmussen, T. (1997) Social interaction and the new media: the construction of communicative contexts, Nordicom-Information, 2-3, pp. 1-12. Revisado: setiembre 12 de 2012. Disponible:

http://www.nordicom.gu.se/common/publ_pdf/5_001_012.pdf

Schneider, F., Feldmann, A., Krishnamurthy, B. \& Willinger, W. (2009)

Understanding Online Social Network Usage from a Network Perspective, Paper presented at Internet Measurement Conference, Chicago, November. Revisado: Octubre 15 de 2012. Disponible: http://www.inet.tu-berlin.de/fileadmin/fg234/Teaching/SS11/Sem_ SS11/SFKW-UOSNUNP-09.pdf

Thulin, E. \& Vilhelmson, B. (2007) Mobiles everywhere: Youth, the mobile phone, and changes in everyday practice, Young, Vol 15, 3, 235-253 
Vesnić-Alujević, L. (2012) Political communication on Facebook:

A case study of the European Parliament profile page for the elections 2009 Communication Management Quarterly : Časopis za upravljanje komuniciranjem 22, 35-54. Revisado: octubre 12 de 2012. Disponible:

http://www.fpn.bg.ac.rs/wp-content/uploads/CM22-Web.pdf

Zuluaga, J. (2012) Internet: nuevas audiencias, ¿̨nuevos ciudadanos?. En Bonilla, J., Cataño, M., Rincón, O. y Zuluaga, J. De las audiencias contemplativas a los productores conectadados. Bogotá: Universidad de los Andes/Universidad EAFIT/Pontificia Universidad Javeriana, pp. 241-262 